\title{
Tackling SARS-CoV-2 Pandemic: The Way Forward
}

\author{
S. A. Tabish ${ }^{1,2}$ \\ ${ }^{1}$ FRCP, Royal College of Physicians, London, UK \\ ${ }^{2}$ Sher-i-Kashmir Institute of Medical Sciences, Srinagar, India \\ Email: amintabish@gmail.com
}

How to cite this paper: Tabish, S.A. (2020) Tackling SARS-CoV-2 Pandemic: The Way Forward. Health, 12, 1202-1216.

https://doi.org/10.4236/health.2020.129088

Received: August 30, 2020

Accepted: September 22, 2020

Published: September 25, 2020

Copyright (c) 2020 by author(s) and Scientific Research Publishing Inc. This work is licensed under the Creative Commons Attribution International License (CC BY 4.0).

http://creativecommons.org/licenses/by/4.0/

\begin{abstract}
The world is engaged in containing the COVID Pandemic and controlling further spread and deaths. The global spread of the virus has overwhelmed health systems, and caused widespread social and economic disruption. Some nations have done a better job than rest of the world. The global spread of the virus has overwhelmed health systems, and caused widespread social and economic disruption. There is a strong case that COVID-19 could be with us in one way or another until a vaccine comes on the market or herd immunity is achieved. A long crisis, could stretch the international order to its breaking point. The virus has succeeded in confining almost all human beings in their homes. Balancing act between public health crisis and complex societal implications is an inescapable necessity. The pandemic will alter the world forever. An economic slowdown, severe recession, plummeting revenue, increased expenditure, and mental health issues could be the emerging challenges. There will be increased confidence in technology and nations will invest more in public health. We are passing through fragile and critical times in history. People around the world can prevail in response to this extraordinary challenge. Investing in public health, preparedness, and relying on science will bring a better future. Think of one world, one humanity to shape and secure our future.
\end{abstract}

\section{Keywords}

COVID-19, SARS-CoV-2, Pandemic, Economic Impact, State of Children, Preparedness, Vaccine, Surge

\section{Background}

All over the world, the human population faces a grave health-related challenge 
in the form of infectious diseases that are responsible for the highest number of deaths globally. The tiniest primitive invisible life form is now controlling the behavior of the most powerful and evolved life form on earth. Tough times ahead [1] [2]!

Between the years of 1940 and 2020, around 340 new infectious diseases have emerged in the global scenario. The compromised health and disability due to infectious diseases, which accounts for $30 \%$ of all disability-adjusted life years, decreases work productivity and increases morbidity [1] [2]. Since the dawn of civilization, humans and microbes have co-existed and interacted with each other. Is the situation worse now? The answer to this question might be yes. It is because of our immense population and our several activities that lead to flourishing of infections.

\section{Study Design/Extraction Methods}

Review of the existing literature was done, mostly that published in standard medical journals.

Studies were identified by searching electronic databases, scanning reference lists of articles. Explicit, systematic methods are selected to minimise bias, thus providing reliable findings from which conclusions can be drawn and decisions made. COVID-19 term was used to search the databases. SARS-CoV-2 is evolving and much evidence about the treatment is not available, and vaccine development will take its own time, further research is crucial.

\section{Epidemiological Transition}

Modern civilization dates to approximately 10,000 BC. We are consuming earth's resources at a rate that is unsustainable. At around 8000 B.C., the global human population was 5 million. By 1 A.D., the population grew up to 200 million. It reached around 1 billion in the $1800 \mathrm{~s}$ during industrial revolution. The second billion was achieved in only 130 years (1930), the third billion in 30 years (1960), the fourth billion in 15 years (1974), and the fifth billion in only 13 years (1987). During the $20^{\text {th }}$ century alone, the population in the world has grown from 1.65 billion to 6 billion. It is projected to reach 14 to 18 billion by the end of the $21^{\text {st }}$ century [3].

Today, SARS-CoV-2 Pandemic has spread to 216 countries and territories with 30,392,000 people infected, and over 951,300 deaths (as on 18 September 2020) and has officially been termed as a pandemic by WHO on 11 March 2020. Most of the COVID-19-related cases and deaths occurred in the USA, Brazil, India, Russia, Peru, South Africa, Mexico, etc. [3].

Most of the human respiratory infections caused by coronaviruses, including NL63, OC43, HKU1, and hCoV-229E, are mild in nature. However, in the past two decades, two beta-coronaviruses, Middle East respiratory syndrome coronavirus (MERS-CoV) and severe acute respiratory syndrome coronavirus (SARS-CoV), have severely infected more than 10,000 individuals, with mortality rates of $37 \%$ 
and $10 \%$, respectively [4].

The fatality rate of COVID-19 appears to be lower than that of SARS (9.5\%) and Middle East respiratory syndrome (34.4\%), but higher than that of influenza $(0 \cdot 1 \%)[5]$.

\section{Characteristics of SARS-CoV-2}

COVID-19 caused by SARS-CoV-2 has spread rapidly all over the world. The pattern of infection of COVID-19 is unique and unpredictable. Previous studies have suggested that, similar to SARS-CoV, COVID-19 might interact with the angiotensin-converting enzyme 2 receptor in humans. The amino acid sequence of the nucleocapsid (N) protein of COVID-19 is 90\% identical to that of SARS-CoV. COVID-19 might cross-react with the $\mathrm{N}$ protein antibodies of SARS-CoV; however, these antibodies might not provide cross-immunity. In addition, similar to SARS-CoV, COVID-19 (SARS-CoV-2) might inhibit the RNA interference defense mechanism of the host organism [6].

Super-spreaders release significantly higher levels of viral particles compared to other infected individuals, which increases their chances of spreading the infection.

Coronaviruses are enveloped non-segmented positive-sense RNA viruses belonging to the family coronaviridae and order nidovirales. Coronaviruses target the upper respiratory tract, which leads to several ailments, including pneumonia. However, they might also affect central nervous system leading to long-term neurological damage. The RNA genome of coronaviruses encode for sixteen nonstructural proteins (nsp 1 - 16) with known or predicted RNA synthesis and modification functions, and they have been also suggested to trigger evolution of large genomes [7].

Virus characteristics primarily define the risk of infection. These characteristics include the efficacy of the virus to spread, the severity of the disease after infection, and the efficacy and success rates of the available medical resources to contain the spread of the virus. With no vaccine or medication, community intervention becomes the most important response strategy against the pandemic. In a nutshell, COVID-19 risk can be defined as the risk of exposure versus risk of serious illness and death.

SARS-CoV-2 presents six strains. Mutation rate has remained low. In European countries, strain $G$ is widespread. L strain from Wuhan is disappearing gradually. The general risk factors of COVID-19 include advanced age and suffering from other chronic medical conditions such as diabetes, cardiac disorders, etc.

COVID has very fast acceleration and very slow deceleration. Reducing the number of emerging cases to a level where health-care facilities can manage them is important to flatten the curve.

Asymptomatic carriers are persons without clinical symptoms who tested positive for SARS-CoV-2. This infection could result in widespread community 
transmission. Continued measures to protect, screen and isolate infected persons are crucial to contain the pandemic.

\section{Countries Experience}

Countries like Singapore, South Korea and New Zealand, and regions like Hong Kong in China, Taiwan in China moved quickly to contain the transmission of COVID-19. Most countries were unable to contain the initial outbreak and moved to flatten and fight. Leadership of these countries relied on time tested public health principles to protect their citizens from COVID-19.

The COVID-19 pandemic is straining healthcare systems (infrastructure, human, materials and financial resources). It has exposed the weak public health systems and lack of preparedness.

There have been successes as well as failures. The leaders who have opted timely the toughest strategies have been able to decline the highest wave of Pandemic and have pulled out their countries much faster from the pandemic. Leadership and political will make a difference. We must balance quick wins with long-term planning. Balanced realism with optimism is the attitude all global leaders need to adopt. With proper focus on short term wins with long term goals we can win the battle against COVID-19.

\section{Indian Scenario}

India with over 1.38 billion population has to have robust and evidence-based health care management and action plans in place with extraordinary monitoring, supervision and continuous assessment in place to control this pandemic. India has 0.9 hospital beds/1000 population as compared to 13.4 in Japan and 13.2 of South Korea and 3.9 in UK and 301 in Sri Lanka. India has 2.3 ICU beds/100,000 population as compared to 34.2 in USA, 29.2 in Germany, and 306 in China. India has 0.8 physicians/ 1000 population as compared to 4.1 in Italy, 2.3 in Singapore and 2.8 in UK. Similarly, India has 2.1 nurses/1000 population as compared to 7.2 in Singapore, 3 in Thailand and 3.4 Global average. A rough estimate of India having 30,000 ventilators is highly inadequate for a huge population of 1.38 billion [8] [9] [10].

There is no immunity against COVID-19 and the entire population is at risk of contracting it if exposed. COVID-19 has Ro of 2.2 (one infected person shall, on an average infect another 2.2 if not isolated). The doubling time of cases is 7 10 days. If containment is not done, it might infect $30 \%-40 \%$ of the population, out of which $5 \%$ shall require hospitalisations, and $1 \%$ ICU care. In worst scenarios, India will have 152 million people requiring medical care, 41.4 million hospitalisations with 12 million in ICU [11] [12].

Some States in India are experimenting Containment Zones. The Cluster Containment Strategy would be to contain the pandemic within a defined geographic area by early detection of cases, breaking the chain of transmission and thus preventing its spread to new areas, can be considered. In Bhilwara Model, a com- 
plete curfew, aggressive follow-up with all in the neighbourhood, large-scale testing, and prevention of travel is required. To prevent further spread of COVID-19, geographical quarantine followed by cluster containment is the better way to go. It is also more flexible. Intensive risk communication campaign will be followed to encourage all persons to stay indoors for an initial period of 28 days, to be extended based on the risk assessment. Red, Orange and Green-the colour code for traffic lights will now be used on the country's map to COVID-19 and virus-free zones, and therefore people's movement. There must be more aggressive restriction in hotspots (lockdown, social distancing). Lifting restrictions should be done in a staggered manner otherwise it can lead to a deadly resurgence. COVID-19 in India is now growing at the fastest rate in the world (nearly 100,000 cases a day). India is far short of resources required to impede the pandemic. Almost 41 percent of the population lack basic handwashing facility. India's health system cannot handle the upcoming turmoil, and the Government has done a great job containing the virus till now. This time should be used for consolidating patient-level data (clinical, labs, images, outcomes) from cases around the world. This could lead to three potential applications-population-level predictions, patient-level predictions of outcomes, and quicker and more effective diagnostics [9] [10] [11].

\section{The US Response}

COVID-19 is a disaster for Americans. As on today, it has more cases and deaths than other countries. The American government's inaction has allowed the virus to spread inside its borders, and it has actively increased the risk to other countries. A faulty diagnostic kit rolled out by the American Centre for Disease Control \& Prevention and slow approvals for other testing kits led to the disease spreading under the nose of the administration. The key problem with the kits is what's known as a negative control. CDC's test uses the polymerase chain reaction (PCR) assay to find tiny amounts of the SARS-CoV-2 genome in, say, a nose swab. To make sure a test is working properly, kits also include DNA unrelated to SARS-CoV-2. The assay should not react to this negative control, but the CDC reagents did at many, but not all, state labs [13].

Because of slow initial response to the pandemic, tens of thousands more people could die. Millions more could lose their jobs, if the situation is not brought under control on a war-footing. Social distancing and lockdown are an inescapable necessity. It took the US 99 days to hit 1 million, 43 days to get to 2 million, and 28 days to reach 3 million. Fifteen days later, it hit the 4 million case mark, and took 17 days more to reach 5 million on 9 August 2020. A high number of deaths above normal in the US (over 200,000 in mid-August) is higher than it would be in a normal year. It is argued that the official death counts may be substantially underestimating the overall effects of the coronavirus. Amid Pandemic, nearly $11 \%$ of adults in the US said they had seriously considered suicide in the previous 30 days as the coronavirus pandemic takes a toll on Americans. 


\section{Surge in COVID19 Cases}

New COVID-19 cases have been spiking from India to South Africa to Mexico in a clear indication that the pandemic is far from over, while India and Brazil now sit behind only the United States in the number of reported infections.

As of 17 September 2020, India has 5,118,300 infections and 82,245 deaths. Brazil reported 4,421,700 cases and 134,180 deaths. Russia reported a steady rise in new infections during the last few weeks and new hot spots have emerged across the vast nation of about 147 million. Russia's case load is fourth among nations with 1,085,300 cases and 19,061 deaths US, which has seen 6,823,400 infections and over 201,350 deaths [3].

It took 72 days for the world to register the first 1 million COVID-19 cases. The rate of growth in adding new cases has been ever increasing. COVID-19 cases in India have grown from 1 million to 4 million in 50 days. The same rise took 75 days in Brazil and 86 in the US. On 4 September 2020, India's tally went from 3 million to 4 million in just 13 days, the fastest 1 million rise yet. India crossed 5 million cases on 15 September. There has been a steep exponential rise in recoveries-from 50,000 in May to 300,000 in September.

The US currently accounts for the world's highest number of infections and fatalities. The country is now having 40-plus thousand new cases a day likely to go up to 100,000 a day if this does not turn around. The recent surge in the number COVID-19 cases across the US has resulted in delays in processing and delivering test results. The soaring demand for COVID-19 molecular diagnostic tests across the US is slowing the time in which test results can be provided. This demand is primarily due to the rapid, continuing spread of COVID-19 infections across the nation. Despite that dramatic increase, demand for testing is increasing even faster.

India accounts for the world's second highest number of cases. Nearly 100,000 new cases are added every day. The surge in COVID-19 testing, now available more extensively and at a reasonable price, could be a major factor. India now has 1026 diagnostic labs (741 in the government sector and 285 private labs). The COVID-related health infrastructure has been strengthened with the availability of 1039 dedicated COVID Hospitals with 1,76,275 isolation beds, 22,940 ICU beds and 77,268 oxygen-supported beds. Moreover, 8958 COVID Care Centres with 8,10,621 beds with 1,39,483 isolation beds, 11,539 ICU beds and 51,321 oxygen-supported beds have been operationalised. Initially there were nearly 100 laboratories. The per day testing capacity which was around 140,000 on May 25 has been increased to 1000,000 (over 20,000 tests per million residents) per day now. Another factor thought to be responsible for the exponential rise in cases is the high reproduction number of the virus [the number of cases, on average, an infected person will cause during their infectious period]. The numbers are doubling at such a fast rate due to factors such. Third factor is that with the lifting of lockdown norms people's behaviour has changed again as they are not acting as responsibly as before in terms of social distancing, and testing 
has become more liberal.

\section{Management of SARS-CoV-2 Infection}

Clinical diagnosis is done based on symptoms, chest imaging, etc. However, the infection can be confirmed only by genetic testing of samples obtained from the respiratory tract (such as throat swabs). Due to absence of appropriate antiviral therapy, the patients are kept on supportive care [14]. The suspected individuals must be isolated, until confirmation. If they are confirmed to be positive, then they must be quarantined. Furthermore, since asymptomatic transmission is also possible, it is necessary to test all the persons who have been in the areas with known transmission. In addition, the symptomatic mothers who are still breastfeeding must maintain proper hygiene before and after handling of the baby.

In case of shortage of hospital beds, the infected individuals who are not severely ill could be isolated and treated at home itself. No hospitalization is needed unless the symptoms worsen. This strategy could be employed to save the hospital capacity for the more needy people and prevent unintended infections to the otherwise healthy individuals while they await their test results at the hospital.

Accurate and scalable point-of-care (POC) tests for the diagnosis of COVID-19 would increase the scope for diagnosis to be made in the community and outside the laboratory setting [15] [16]. They would have the potential to reduce the time to obtaining an actionable result, could support early identification of those with COVID-19 and could also support appropriate use of isolation resources, infection control measures, and recruitment into clinical trials of treatments. An increasing number of diagnostic devices that are potentially suitable for the diagnosis of COVID-19 at point-of-care are in development. Different devices may be more suitable for diagnosing new cases on infection, while others, especially those that test for the presence of antibodies, are better suited to determining whether an individual has previously been infected [17].

South Korea has shown an exemplary performance by significantly slowing the infection spread in the country with a population of 50 million without any lockdowns or other severe measures that were adopted by China. It has demonstrated that diagnosis at large scale is key to epidemic control, along with case isolation and contact tracing [18].

A speedy recovery would depend on most of the world getting the pandemic under control or achieving herd immunity.

Timely detection of hypoxia and availability of Pulse Oximeters can prevent death. The first trigger is silent hypoxia. In these patients, microvasculitis occurs in lungs, microclots are formed due to vascular endothelial dysfunction with resultant intussusception of the artery i.e. the artery is partially thrombosed and partially patent so perfusion is maintained. Such patients may be given one dose of antiviral (remdesivir), one dose of LMWH (low molecule weight heparin), water-soluble aspirin stat and home oxygen therapy before shifting to a hospital. 
Patients with high ESR, CRP (>26), ferritin and d-dimer levels are at high risk for severe illness. If lymphocyte count is $<1000$ patient should be admitted in a hospital and if progressive lymphopenia $(<800)$ with rising $\mathrm{LDH}$, patient needs admission in ICU.

Glucocorticoids may reduce mortality compared with standard care. The effectiveness of most interventions is not definite and evidence-based. Post-acute SARS-CoV-2 disease involves multisystem. Many patients recover spontaneously. Home pulse oximetry helps.

COVID-19 is likely to be more fatal in older, male, overweight patients with comorbidities. Moreover, patients with severe lung, liver or renal involvement have greater mortality.

\section{Economic Impact}

Today we are confronted with an unprecedented crisis world has never experienced before. The global economy has hit the worst recession because of the pandemic. COVID-19 pandemic has been one of the greatest global collective failure. The pandemic and society's responses to it are hitting economies across the world simultaneously, suffering both a demand and a supply shock. The worst economic fallout since the Great Depression is anticipated. The International Monetary Fund (IMF) on April 14, 2020 slashed growth forecast for the Indian economy, projecting a GDP growth of 1.9 percent in 2020 [10] [11] [12].

The COVID-19 has forced many around the world to rethink their daily lives, from work to school to entertainment. In response to travel bans, school closures, and social distancing recommendations to limit the spread of the virus, many people have turned to digital tools to keep some semblance of normality. According to the International Labour Organization, the labor and economic crisis induced by the COVID-19 pandemic could lead to a rise in global employment from 5.3 million to 24.7 million. Overall, the global impact has been predicted to be in the range of $\$ 77$ billion to $\$ 347$ billion or $0.1 \%$ to $0.4 \%$ of global GDP [19].

According to the UN's trade and development agency, UNCTAD, apart from the tragic human consequences of the COVID-19 coronavirus epidemic, the economic uncertainty it has sparked will likely cost the global economy $\$ 2$ trillion in 2020. Heavily indebted developing countries, particularly commodity exporters, face a particular threat. There is a need to take urgent steps to reduce the economic impact [20].

In the long-term the pandemic will reduce the productive capacity of the global economy. Greater moves toward selective self-sufficiency can be anticipated given supply chain vulnerability.

\section{State of Children}

Covid-19 pandemic created largest disruption of education in history. Over 1.6 billion learners across the world have been affected by the disruption of the education system, low income countries are worst affected exacerbate existing 
disparities. According to United Nations, nearly 24 million children are at risk of not returning to school next year due to the economic fallout of COVID-19. UNESCO estimates that 23.8 million additional children and youth [from pre-primary to tertiary] may drop out or not have access to school next year due to the pandemic's economic impact alone. COVID-19 could cause hundreds of thousands of additional child death. An estimated 42 - 66 million children could fall into extreme poverty because of the crisis this year, adding to the estimated 386 million children already in extreme poverty in 2019. The looming global recession resulting from the outbreak could cause hundreds of thousands of additional child deaths this year, effectively reversing recent gains in reducing infant mortality, and push millions of children into extreme poverty. However, some children are destined to bear the greatest costs. Among those who will be badly hit are children living in slums, refugee and displacement camps, and zones of active conflict. All children have a right to survive, thrive and fulfil their potential to the benefit of a better world.

According to UNICEF report, practically all students worldwide are now out of school because of the pandemic. 2 in 5 schools around the world lacked basic handwashing facilities prior to the COVID-19 pandemic. Nearly 190 countries have imposed school closures, affecting 1.5 billion children and young people. The losses in learning today, and in their future development, are hard to fathom. Child nutrition is another vital concern. With classrooms shuttered, the nearly 310 million children worldwide who rely on school meals are missing out on this daily dose of nutrition. There is also a danger that girls will drop out of school, leading to an increase in teenage pregnancies [21].

Another concern is delay in routine immunization of children that makes them vulnerable to disease. Once the pandemic is over, every child in need must be vaccinated. Uunprecedented international solidarity is crucial for children and humanity [10] [11] [12].

\section{Emerging Perspectives}

As scientists strive to understand the respiratory COVID-19 disease and the virus that causes it, the need for aggressive and fruitful research on the subject is the need of the hour.

Severely ill patients must be first hospitalized. The individuals with moderate symptoms must be sent to repurposed facilities for basic medical support. If they are tested negative twice, then they are released. The individuals with minimal symptoms are advised to quarantine themselves for two weeks. Planning for surge capacity and the necessary equipment and staff in ICUs has to be done on a top priority basis. Doctors and nurses have to be trained in ICU care. Ventilation strategy should be applied to each patient's mechanical lung properties. Triage decision system must be in place. Optimization of supportive care is crucial. Lung injury with features of ARDS (Acute Respiratory Distress Syndrome) is the main characteristic of SARS-CoV-2 infection. Awake prone positioning is 
emerging as a standard care for SARS-CoV-2 infection, the objective in typical ARDS is to reduce ventilation/perfusion mismatching, hypoxemia and shunting. Awake prone position appears to be safe.

Several published reports of early clinical descriptions of COVID-19 have emerged from Hubei province in China, and many more will come. These early reports, typically simple descriptive case series of patients hospitalized with COVID-19 (mostly with pneumonia), provide valuable information on the more severe end of the disease spectrum. The current best estimate is that about $81 \%$ of people with COVID-19 have mild disease and never require hospitalization. These cases have not yet featured much in published clinical descriptions [22].

\section{Impact \& Implications}

COVID-19 can be contained by effective measures like interrupting human-tohuman transmission including reducing secondary infections among close contacts and health care workers, preventing transmission amplification events, identifying, isolating and caring for patients early, including providing optimised care for infected patients, focusing on treatment options, and accelerating the development of diagnostics, therapeutics and vaccines and communicating critical risk and event information to all communities and counter misinformation.

The strategy for stopping the transmission includes: travel restrictions, social distancing, test, track, treat, isolate: surveillance and response, map and engage health infrastructure, personnel, stock PPE, other essential supplies, risk communication, community engagement, research \& development. Contact tracing is crucial. Prolonged stays at home are inevitable. While disrupting the work and educational engagements of millions, it might induce affective and psychological distress if younger and active people are not gainfully engaged. People are going to rely heavily on visual media and social media platform for news and entertainment. This is the high time to provide some cash in the hands of public who are not only facing immense uncertainty on the health front but are directly glaring at a widespread loss of livelihoods.

\section{Advances in Anti-Viral Antibody Detection}

Antibody detection involves the use of blood or plasma samples to determine the presence of anti-SARS-CoV-2 antibodies-IgM and/or IgG. IgM becomes detectable a few days post-infection and lasts for a couple of weeks, after which it switches to IgG, making IgM an earlier indicator of SARS-CoV-2 infection and IgG a slightly later indicator of current or prior infection. Neutralization assays are the classic method used to determine the level of anti-viral antibodies present in a patient sample required to inhibit virus infection in cell culture conditions. Such testing requires Biosafety Level 3 laboratory facilities, but the ability to determine the strength of antibodies capable of inhibiting viral infection is crucial for both short-term therapeutics and long-term vaccine development [23]-[30]. 
New Cochrane review shows that antibody tests could have a useful role in detecting if someone has had COVID-19, but that timing is important. The tests were better at detecting COVID-19 in people two or more weeks after their symptoms started, but we do not know how well they work more than five weeks after symptoms started. The immune system of people who have COVID-19 responds by developing proteins in the blood called antibodies that attack the virus. Detecting antibodies in people's blood may indicate whether they currently have COVID-19 or have had it previously [31].

\section{Search for Effective Treatments}

Many of the drugs being developed or tested for SARS-CoV-2 are antivirals. These would target the virus in people who already have an infection. Antivirals work better if you administer them sooner, before the virus has a chance to multiply significantly or cause damage to the lungs or other tissues. Some antivirals include Remdesivir, Arbidol, EIDD-2801, Favipiravir, Kaletra (a combination of two drugs-lopinavir and ritonavir), Merimepodib (VX-497) [23]-[30].

Scientists are also looking at other ways to target the virus or treat the complications of COVID-19. Among them is ibuprofen. Scientists in the United Kingdom reported success in initial tests with a protein called interferon beta. The protein is produced by the body during viral infections. The protein is inhaled directly into the lungs of someone infected with the novel coronavirus in hopes of stimulating an immune response. The antibodies are collected from people hospitalized with COVID-19. If proven safe and effective, the antibodies would be given via blood transfusions to people newly infected with the virus.

Monoclonal antibodies trigger the immune system to attack the virus. Like antibodies made by the body's immune system, these laboratory-made molecules target a specific invader, such as SARS-CoV-2.

Treatment with high dose methylprednisolone and tocilizumab is associated with faster respiratory recovery and lower likelihood of mechanical ventilation. This short-ccourse combination therapy suppresses COVID-19 Cytokine Storm (a complication of severe acute respiratory syndrome SARS-CoV-2 infection). A word of caution is that routine use of systemic corticosteroids should be avoided.

Pyronaridine and Artesunate are potential antiviral drugs against COVID-19. Favipivarir too is recommended for new recurrent flu.

\section{Vaccine Development}

There are 139 vaccines in development and 26 in human trial. Some of the projects include Moderna, Inovio, University of Oxford in England, University of Queensland in Australia, Pharmaceutical companies (Johnson \& Johnson and Sanofi are both working on a vaccine of their own). Pfizer has also teamed up with German biotech company BioNTech to develop a vaccine. Scientists at CanSino Biologics in China are also working on a potential vaccine. Advances in genetic sequencing and other technological developments have sped up some of 
the earlier laboratory work for vaccine development. Some researchers are of the opinion that a "human challenge trial" could speed up the vaccine clinical trials-potentially shaving months off the timeline. Potential vaccines have to pass through the same clinical trial stages [24] [25].

Researchers from China reported that phase 1 of the first ever human trial of a SARS-CoV-2 vaccine had concluded in Wuhan. This vaccine goes by the name of a "recombinant adenovirus type-5 (Ad5) vectored COVID-19 vaccine.

The Oxford adenovirus COVID-19 vaccine is safe and elicits significant immune responses in study volunteers. The vaccine, which the researchers call ChAdOx1 nCoV-19 but many refer to as the Oxford vaccine, uses a weakened adenovirus from chimpanzees. Phase III trials are currently underway [24].

Russia on 11 August 2020 became the first country to officially register a COVID-19 vaccine (Sputnik V) and declare it ready for use. According to Russian President, the vaccine underwent the necessary tests and has proven efficient, offering a lasting immunity from the coronavirus. Production of vaccine has already started. Researcher and scientists at home and abroad are sceptical and argue that the rush to start using the vaccine before Phase 3 trials could backfire.

Even if there is a breakthrough in vaccine development by researchers, it will not be easy to manufacture nearly 8 billion doses in a short period. The logistics like vials and cold chain for transportation and storage will be a much bigger challenge. Affordability will be another factor.

\section{Learning from Tigers}

There have been major success stories from China, New Zealand, South Korea, Taiwan in China, Hong Kong in China and Japan. Transmission dynamics of any outbreak are inherently contextual. China followed a policy of meticulous case and contact identification. In Wuhan more than 1800 teams of epidemiologists, with a minimum of 5 people/team, were tracing tens of thousands of contacts a day. Contact follow up was practiced with a high percentage of identified close contacts completing medical observation. New technologies were applied such as the use of big data and artificial intelligence to strengthen contact tracing and the management of priority populations. China's robust and dynamic mechanism to contain the rapid spread of virus has changed the course of a rapidly escalating and deadly epidemic. The SARS-CoV-2 virus is capable of causing enormous health, economic and societal impacts in any setting. To reduce disease and save lives it is crucial to build scenarios and strategies only on the basis of well-known pathogens risks failing to exploit all possible measures to slow transmission of the virus. China's uncompromising and rigorous use of nonpharmaceutical measures to contain transmission of the virus in multiple settings provides vital lessons for rest of the world. China's unprecedented public health response in China reversed the escalating cases in both Hubei, where there has been widespread community transmission, and in the importation 
provinces, where family clusters appear to have driven the outbreak. Measures required to succeed in containment of transmission needs an unusual and unprecedented speed of decision-making by top leaders, operational thoroughness by public health systems, and engagement of society. The time gained by rigorously applying containment measures should be used more effectively to rapidly develop the specific tools that are needed to ultimately stop this virus. It is crucial to maintain an appropriate level of emergency management protocols, depending on the assessed risk in each area and recognizing the real risk of new cases and clusters of COVID-19 as economic activity resumes, movement restrictions are lifted, and schools reopen [32].

\section{Conclusion}

In a world of 7.8 billion people, countries have to be in a state of emergency preparedness to tackle emerging infectious diseases. It needs an aggressive and focused action, with coordination among all departments of the governmenthealth, security, finance, commerce, transport, trade, information, diplomacy, etc. The approach to tackle this pandemic has to be solidly evidence-informed, robust, pragmatic and effective. Creating awareness about wearing mask, social distancing, frequent handwashing, robust contact tracing, providing quality healthcare to COVID-19 patients, availability of quality PPE to health professionals and looking after weaker sections of society are crucial to flatten the curve of pandemic. It needs political will. Balancing act between public health crisis and complex societal implications is an inescapable necessity. Scientists must find an effective therapy on a war footing. During these most difficult times in human history, nations need to ensure that people can meet their needs and that businesses can pick up once the acute phases of the pandemic pass. Social unrest and food riots must be pre-empted. Nations will have to prioritise spending. Special attention needs to be paid towards education and mental health of children and young adults. People around the world can prevail in response to this extraordinary challenge. Investing in public health, preparedness, and relying on science will bring a better future. The world will emerge resilient from COVID pandemic if the crisis prompts a radical reset of our economic and social life with policies that invest in people with the objective of one humanity, one world.

\section{Conflicts of Interest}

The author declares no conflicts of interest regarding the publication of this paper.

\section{References}

[1] Tabish, S.A. (2009) Recent Trends in Emerging Infectious Diseases. International Journal of Health Sciences, 3, 5-8.

[2] Tabish, S.A. and Nabil, S. (2015) The Future of Humanity and Microbes: Impact of Emerging Infectious Diseases on Global Health Economies. International Journal of 
Science and Research, 4, 2427-2442.

[3] Worldometer. World Population. https://www.worldometers.info/world-population

[4] Cascella, M., Rajnik, M., Cuomo, A., Dulebohn, S.C. and Di Napoli, R. (2020) Features, Evaluation and Treatment Coronavirus (COVID-19). https://www.ncbi.nlm.nih.gov/books/NBK554776

[5] Fauci, A.S., Lane, H.C. and Redfield, R.R. (2020) Covid-19-Navigating the Uncharted. The New England Journal of Medicine, 382, 1268-1269.

https://doi.org/10.1056/NEJMe2002387

[6] Kannan, S., Shaik Syed Ali, P., Sheeza, A. and Hemalatha, K. (2020) COVID-19 (Novel Coronavirus 2019)-Recent Trends. European Review for Medical and Pharmacological Sciences, 24, 2006-2011.

[7] Denison, M.R., Graham, R.L., Donaldson, E.F., Eckerle, L.D. and Baric, R.S. (2011) An RNA Proofreading Machine Regulates Replication Fidelity and Diversity. RNA Biology, 8, 270-279. https://doi.org/10.4161/rna.8.2.15013

[8] Tabish, S.A. (2020) COVID-19: An Unprecedented Crisis That Needs an Extraordinary Response. Archives of Community Medicine and Public Health. https://doi.org/10.17352/2455-5479.000075

[9] Tabish, S.A. (2020) COVID-19 Pandemic: The Crisis and the Longer-Term Perspectives. Journal of Cardiology \& Current Research, 13, 41-44.

[10] Tabish, S.A. (2020) COVID-19 Pandemic: Emerging Perspectives \& Future Trends. Journal of Public Health Research, 9, 1785. https://doi.org/10.4081/jphr.2020.1786

[11] Tabish, S.A. (2020) COVID 19 Pandemic: Calm before Storm. IJSR, 9, 175-179. https://doi.org/10.4081/jphr.2020.1786

[12] Tabish, S.A. (2020) Life and Livelihood during the COVID 19 Pandemic. Journal of Clinical Immunology \& Immunotherapy, 6, 035. https://doi.org/10.24966/CIIT-8844/1000035

[13] Cohen, J. (2020) The United States Badly Bungled Coronavirus Testing-But Things May Soon Improve. Sciencemeg.org. https://doi.org/10.1126/science.abb5152

[14] Wu, Z. and McGoogan, J.M. (2020) Characteristics of and Important Lessons from the Coronavirus Disease 2019 (COVID-19) Outbreak in China: Summary of a Report of 72314 Cases from the Chinese Center for Disease Control and Prevention. $J A M A, 323,1239-1242$. https://doi.org/10.1001/jama.2020.2648

[15] Wang, C., et al. (2020) A Novel Coronavirus Outbreak of Global Health Concern. The Lancet, 395, 470-473. https://doi.org/10.1016/S0140-6736(20)30185-9

[16] Nguyen, T., et al. (2019) Novel Coronavirus Disease (COVID-19): Paving the Road for Rapid Detection and Point-of-Care Diagnostics. Micromachines, 11, 306. https://doi.org/10.3390/mi11030306

[17] Green, K., Graziadio, S., Turner, P., Fanshawe, T. and Allen, J. (2020) Molecular and Antibody Point-of-Care Tests to Support the Screening, Diagnosis and Monitoring of COVID-19. Cebm.net.

[18] Normile, D. (2020) Coronavirus Cases Have Dropped Sharply in South Korea. What's the Secret to Its Success? https://doi.org/10.1126/science.abb7566 https://www.sciencemag.org/news/2020/03/we-are-social-species-how-will-social-di stancing-affect-us

[19] Abiad, A., Arao, R.M., Dagli, S., et al. (2020) The Economic Impact of the COVID19 Outbreak on Developing Asia. Asian Development Bank, Mandaluyong. 
https://doi.org/10.22617/BRF200096

[20] World Economic Forum and United Nations (2020) This Is How Much the Coronavirus Will Cost the World's Economy, According to the UN.

https://www.weforum.org/agenda/2020/03/coronavirus-covid-19-cost-economy-20 20-un-trade-economics-pandemic

[21] United Nations (2020) UN Policy Brief. The Impact of COVID 19 on Children. https://www.un.org/sites/un2.un.org/files/policy_brief_on_covid_impact_on_childr en_16_april_2020.pdf

[22] Zhou, F., Yu, T., Du, R., et al. (2020) Clinical Course and Risk Factors for Mortality of Adult Inpatients with COVID-19 in Wuhan, China: A Retrospective Cohort Study. The Lancet, 395, 1054-1062.

[23] Bruning, A.H.L., Leeflang, M.M.G., Vos, J.M.B.W., Spijker, R., de Jong, M.D., Wolthers, K.C., et al. (2017) Rapid Tests for Influenza, Respiratory Syncytial Virus, and Other Respiratory Viruses: A Systematic Review and Meta-Analysis. Clinical Infectious Diseases, 65, 1026-1032. https://doi.org/10.1093/cid/cix461

[24] Liu, Y., Liu, Y., Diao, B., Ren, F.F., et al. (2020) Diagnostic Indexes of a Rapid IgG/IgM Combined Antibody Test for SARS-CoV-2.

https://doi.org/10.1101/2020.03.26.20044883

[25] Pan, Y., Li, X., Yang, G., Fan, J., et al. (2020) Serological Immunochromatographic Approach in Diagnosis with SARS-CoV-2 Infected COVID-19 Patients.

https://doi.org/10.1101/2020.03.13.20035428

[26] Li, Z., Yi, Y., Luo, X., Xin, N., et al. (2020) Development and Clinical Application of a Rapid IgM-IgG Combined Antibody Test for SARS-CoV-2 Infection Diagnosis. Journal of Medical Virology, 92, 1518-1524.

[27] Lin, D., Liu, L., Zhang, M., Hu, Y., et al. (2020) Evaluation of Serological Tests in the Diagnosis of 2019 Novel Coronavirus (SARS-CoV-2) Infections during the COVID-19 Outbreak. https://doi.org/10.1007/s10096-020-03978-6

[28] Wölfel, R., Corman, V., Guggemos, W., Seilmaier, M., Mueller, M., Niemeyer, D., et al. (2020) Virological Assessment of Hospitalized Patients with COVID-2019. Nature, 581, 465-469. https://www.nature.com/articles/s41586-020-2196-x https://doi.org/10.1038/s41586-020-2196-x

[29] Cochrane Review. Antobody Tests for Identification of Current and Past Infections with SARS-CoV-2: How Accurate Antibody Tests Are for Detecting COVID-19. https://www.cochrane.org/news/new-cochrane-review-assesses-how-accurate-antib ody-tests-are-detecting-covid-19

[30] Calina, D., Docea, A.O., Petrakis, D., Egorov, A.M., et al. (2020) Towards Effective COVID-19 Vaccines: Updates, Perspectives and Challenges (Review). International Journal of Molecular Medicine, 46, 3-16. https://doi.org/10.3892/ijmm.2020.4596

[31] Bedford, J., Enria, D., Giesecke, J., Heymann, D.L, Ihekweazu, C., Kobinger, G., et al. (2020) COVID-19: Towards Controlling of the Pandemic. The Lancet, 395, 1015-1018. https://doi.org/10.1016/S0140-6736(20)30673-5

[32] WHO (2020) Report of the WHO-China Joint Mission on Coronavirus Disease 2019 (COVID-19).

https://www.who.int/docs/default-source/coronaviruse/who-china-joint-mission-o n-covid-19-final-report.pdf 